Postprint of Critical Reviews in Food Science and Nutrition Volume 56, 2016 - Issue 8, Pages 1242-1252

DOI: $10.1080 / 10408398.2012 .761173$

\title{
1 Amino Acid Degradations Produced by Lipid Oxidation Products
}

2 FRANCISCO J. HIDALGO and ROSARIO ZAMORA

3 Instituto de la Grasa, Consejo Superior de Investigaciones Científicas, Avenida Padre

4 García Tejero 4, 41012 Seville, Spain

5

6

7

8

9

10

11

12

13

14

15

16

17 Footnote to title page: Address correspondence to Rosario Zamora, Instituto de la

18 Grasa, Avenida Padre García Tejero 4, 41012 Seville, Spain. Tel.: +34 954611550.

19 Fax: +34 954616 790. E-mail: rzamora@ig.csic.es 
21 Differently to amino acid degradations produced by carbohydrate-derived reactive

22 carbonyls, amino acid degradations produced by lipid oxidation products are lesser

23 known in spite of being lipid oxidation a major source of reactive carbonyls in food.

24 This article analyzes the conversion of amino acids into Strecker aldehydes, $\alpha$-keto

25 acids, and amines produced by lipid-derived free radicals and carbonyl compounds, as

26 well as the role of lipid oxidation products on the reactions suffered by these

27 compounds: the formation of Strecker aldehydes and other aldehydes from $\alpha$-keto acids;

28 the formation of Strecker aldehydes and olefins from amines; the formation of shorter

29 aldehydes from Strecker aldehydes; and the addition reactions suffered by the olefins

30 produced from the amines. The relationships among all these reactions and the effect of

31 reaction conditions on them are discussed. This knowledge should contribute to better

32 control food processing in order to favor the formation of desirable beneficial

33 compounds and to inhibit the production of compounds with deleterious properties.

34 Keywords acrylamide, carbonyl-amine reactions, flavor formation, lipid oxidation,

35 Maillard reaction, Strecker aldehydes, styrene 
Amino acid catabolism by microorganisms in foods has long received a considerable attention mostly as a consequence of its repercussions on food quality and safety (Ardö, 2006; Fernández and Zúñiga, 2006). Thus, it was early known that both pleasant and unpleasant aroma compounds were produced as a consequence of amino acid degradations (Urbach, 1993). Moreover, amino acids are well known precursors of compounds such as biogenic amines that may compromise the health of consumers (Marcobal et al., 2012). thermal processing in food products (Taeymans et al., 2004; Zhang et al., 2009).

Differently to amino acid degradations produced by carbohydrate-derived reactive carbonyls, amino acid degradations produced by lipid oxidation products are lesser known in spite of being lipid oxidation a recognized source of reactive carbonyls in

57 foods (Cheng, 2010; Choe and Min, 2006). Furthermore, chemical reactivity of lipid- 
carbonyls, and both types of carbonyl compounds usually produce very similar nonenzymatic browning reactions (Zamora and Hidalgo, 2005).

In an attempt to update the diverse studies carried out in recent years in this field, this article reviews the amino acid degradations produced by oxidized lipids as well as the reactions in which the compounds produced in these degradations are involved.

\section{STRECKER DEGRADATION OF AMINO ACIDS PRODUCED BY LIPID} OXIDATION PRODUCTS

The Strecker degradation of amino acids is produced by a wide range of lipid oxidation products, including primary, secondary, and tertiary products of lipid oxidation (Zamora et al., 2008). However, the reaction is produced to different extents depending on the lipid oxidation product involved, which is likely a consequence of both the influence of the lipid-derived carbonyl on the reaction pathway and the existence of parallel reactions in which these oxidized lipids may be involved and that compete with the Strecker reaction.

The Strecker degradation of amino acids by oxidized lipids was firstly described in 2004 for the formation of phenylacetaldehyde by phenylalanine degradation in the presence of epoxyalkenals (Hidalgo and Zamora, 2004), and later extended to other lipid-derived reactive carbonyls (Hidalgo et al., 2005; Zamora et al., 2007). A general mechanism for the Strecker degradation of amino acids produced by lipid-derived reactive carbonyls is shown in Figure 1. The first step of the reaction is the formation of an imine (imine A) between the amino group of the amino acid and the carbonyl group of the lipid. This imine suffers then an electronic rearrangement as a consequence of the exit of the proton of the carboxylic group. This electronic rearrangement produces the 
loss of carbon dioxide and the formation of a new imine (imine B), which is the origin of the the Strecker aldehyde after hydrolysis.

At the same time that the amino acid is degraded, the lipid-derived carbonyl is transformed into a new derivative in which the carbonyl group has been converted into an amino group (Figure 2). However, this compound is not usually stable and evolves either into polymers or into more stable heterocyclic structures. Thus, the formation of 2-alkylpyridines is usually observed in the reaction of epoxyalkenals and alkadienals with amino acids (Hidalgo and Zamora, 2004; Zamora et al., 2007). On the contrary, 2alkylpyrroles were identified in the reaction of hydroxyalkenals and oxoalkenals with amino acids (Hidalgo et al., 2005; Zamora et al., unpublished).

Although short-chain volatile compounds have received much more attention than long-chain lipid oxidation products, these last compounds are usually produced to a much higher extent than short-chain derivatives. Thus, for example, the Fe(II)/Fe(III)catalyzed linoleic acid hydroperoxide decomposition produced volatiles comprising less than 5 mol\% of total products (Grosch, 1976). For that reason, the ability of long-chain lipid oxidation products, in which the carbonyl group is usually a ketone group, to convert amino acids into their corresponding aldehydes was also investigated. Two different lipid oxidation products were assayed: conjugated epoxyoxooctadecenoates (Zamora et al., 2005) and conjugated oxooctadecadienoates (Zamora et al. 2007) [general structures for these compounds are shown in Figure 2]. According to the obtained results, the ability of aldehydes and ketones for degrading amino acids was similar in most experiments and differences found might be more related to the different solubility of the lipid oxidation products assayed than to a different reactivity. In this case, the reaction also followed a reaction pathway similar to that shown in Figure 1. The only difference was that the carbonyl group was in the middle of a chain, and, 
therefore, the heterocyclic derivative that was produced after the reaction was slightly different. Thus, both epoxyoxooctadecenoates and oxooctadecadienoates were converted in fatty acid chains containing a pyridine ring in the middle of the chain (Figure 2). Consequently, this reaction provides a route for the formation of long chain heterocyclic fatty acid derivatives in food products. Although heterocyclic fatty acid derivatives are not usually determined, their formation (or their syntheses) has been objective of diverse studies (see, for example, Fürmeier and Metzger, 2003; Gardner et al., 2000; Hidalgo and Zamora, 1995).

Differently to secondary and tertiary lipid oxidation products, the primary products of lipid oxidation (the hydroperoxides) do not have a carbonyl group. However, they are easily decomposed to produce both free radicals and carbonyl compounds (Gardner, 1989). The carbonyl compounds produced in this decomposition are the secondary and tertiary lipid oxidation products discussed above, which produce the Strecker degradation of the amino acids by the mechanism indicated in Figure 1. However, this cannot be the unique mechanism by which lipid hydroperoxides degrade amino acids because carbonyl compounds are only a part of the secondary and tertiary lipid oxidation products formed (Gardner, 1989; Reis and Spickett, 2012), and the yields obtained for the formation of Strecker aldehydes by reaction with the hydroperoxides is quite similar to the yields obtained with the lipid-derived reactive carbonyls (Zamora et al., 2008). Therefore, hydroperoxides should have an alternative way to produce the Strecker degradation of amino acids in addition to the reaction of the carbonyls produced as a consequence of their decomposition. This alternative pathway is likely a free radical degradation analogous to that collected in Figure 3. Lipid hydroperoxides are decomposed by means of different agents, including heat and metal traces, to produce the corresponding alkoxyl radicals (Girotti, 1998; Kasaikina et al., 2006; Pazos, 
et al., 2008; Ueda et al., 1996). These radicals can then abstract a proton from the amino acid so that the radicals are converted into hydroxy acids (usual products in these reactions) at the same time that an amino acid radical is produced. This radical (a oxygen-centered radical has been drawn, but other radicals are also possible) suffers then an electronic rearrangement with the loss of carbon dioxide and the exit of a proton, which may react with a new hydroxyl radical to produce the corresponding hydroxy acid. The product of this electronic rearrangement is an imine that is the responsible for the phenylacetaldehyde formation after hydrolysis. A pathway similar to this has been proposed for the degradation of amino acids observed by pyrolysisGC/MS in the absence of carbohydrates (Chu and Yaylayan, 2008; Yaylayan and 142 Keyhani, 2001). PRODUCTS

As observed in Figure 1, the trigger for the electronic rearrangement of imine A, which is the key step for the Strecker degradation, is the exit of the carboxylic proton under the acid conditions at which the reaction is produced. However, the exit of the proton at the $\alpha$-carbon of the amino acid would produce the same result without the loss of carbon dioxide. Zamora et al. (2006a) showed that this proton can leave as a function of the electronic effects of the substituents at the $\alpha$-carbon. Thus, long-chain saturated amines, in which the $\alpha$-carbon is joined to an amino group and an alkyl chain, were converted into carbonyl compounds only to a very low extent. However, the yield for benzylamine, in which the $\alpha$-carbon is joined to an amino group and an aromatic ring, increased to $4.3 \%$. Furthermore, when this carbon was trisubstituted, such as in 2- 
phenylglycine methyl ester, in which the $\alpha$-carbon is joined to an amino group, an aromatic ring, and a methoxycarbonyl group, the reaction yield increased to $49 \%$.

Amino acids are usually trisubstituted at the $\alpha$-carbon: they have the amino group, the carboxylic group, and the chain, which usually starts with a methylene group. Therefore, the proton at the $\alpha$-carbon should leave with a relative easiness. In fact, when phenylalanine was incubated overnight with 4,5-epoxy-2-decenal at $37^{\circ} \mathrm{C}$, the corresponding $\alpha$-keto acid was produced to a higher extent than the Strecker aldehyde (Zamora et al., 2006b). The reaction pathway for this reaction is shown in Figure 4. It is analogous to the reaction pathway for Strecker aldehyde formation shown in Figure 1. The only difference is the exit of the proton at the $\alpha$-carbon in the place of the exit of the carboxylic proton. Therefore, the electronic rearrangement of imine A produces the imine $\mathrm{C}$ in the place of the imine B shown in Figure 1. The later hydrolysis of this imine $\mathrm{C}$ is the origin of the $\alpha$-keto acid. At the same time that the $\alpha$-keto acid is produced, the oxidized lipid is modified analogously to the observed for the Strecker aldehyde formation, and the formation of the corresponding pyridine and pyrrole derivatives shown in Figure 2 should also be expected as byproducts of the $\alpha$-keto acid formation.

Although this degradation has been observed for different lipid-derived carbonyls and a similar degradation of methyl 2-phenylglycine by ribose has also been described (Zamora et al., 2006a), no reports have appeared to date regarding to the ability of lipid hydroperoxides to produce this degradation. In the case of hydroperoxides, the ratio between Strecker aldehydes and $\alpha$-keto acids will likely depend on the different ability of alkoxyl radicals to convert amino acids into either heteroatom- or carbon-centered radicals, respectively. 
When phenylalanine is heated at high temperature in the presence of air and lipidderived reactive carbonyls, the formation of benzaldehyde is usually observed in addition to the formation of phenylacetaldehyde [see, for example, Zamora et al. (2007)]. The formation of this aldehyde, having two carbons less than the original amino acid is likely to take place from the corresponding $\alpha$-keto acid or Strecker aldehyde by a reaction pathway similar to that shown in Figure 5. In the presence of lipid-derived reactive carbonyls, amino acids are converted into either $\alpha$-keto acids, which have the same number of carbons than the original amino acid, or Strecker 
under nonoxidative conditions is very low in comparison to the benzaldehyde produced at high temperature and in the presence of air (Zamora et al. unpublished results).

Depending on the reaction conditions, phenylpyruvic acid can also be decarboxylated to phenylacetaldehyde (Zamora et al., 2006b), which can be later oxidized to benzaldehyde according to the reaction pathway collected in Figure 5. However, this mechanism should be easily distinguished from the direct conversion of phenylpyruvic acid into benzaldehyde described in Figure 5 because oxalic acid cannot be produced if the two carbons are not lost in one step.

\section{CONVERSION OF AMINO ACIDS INTO AMINES BY LIPID OXIDATION}

\section{PRODUCTS}

In addition to the above described conversion of the $\alpha$-amino group of amino acids into a carbonyl group, lipid-derived reactive carbonyls are also able to decarboxylate amino acids converting them into the corresponding amines, which are usually known as biogenic amines when they are produced as a consequence of the action of microorganisms (Shalaby et al., 1996). This reaction has lately received a considerable attention because of its potential implication in the formation of acrylamide as a consequence of asparagine degradation in the presence of lipid-derived reactive carbonyls (Capuano et al., 2010; Zamora and Hidalgo, 2008). It was first hypothesized to be produced as an intermediate step in the conversion of phenylalanine into styrene (Hidalgo and Zamora, 2007). Later, it was confirmed in the conversions of both asparagine into 3-aminopropionamide (Hidalgo et al., 2010a) and phenylalanine into $\beta$ phenylethylamine (Zamora et al., 2012a) by action of lipid oxidation products.

The reaction pathway proposed for this reaction is shown in Figure 6. As observed, the reaction is initiated, analogously to the reactions described above, with the 
227 formation of the imine A between the amino group of the amino acid and the carbonyl group of the lipid oxidation product. However, this time neither the exit of the hydrogen at the carboxylic group (Figure 1) nor the exit of the hydrogen at the $\alpha$-carbon (Figure 4) are produced. In this case, the electronic rearrangement of imine A produces a 5oxazolidinone intermediate, which was first identified in model systems of amino acids and simple aldehydes (Aurelio et al., 2003; Tsuge at al., 1987) and, then, in mixtures with aldehydes having a second functional group such as glycolaldehyde (Chu and Yaylayan, 2009). The importance of the 5-oxazolidinone formation in this pathway lies in its ability to decarboxylate and form a non-stabilized azomethine ylide, which is prone to undergo a 1,2-prototropic shift and form two isomeric imines (imines B and D in the figure). As can be observed, the formed imine B is identical to imine B produced in the pathway shown in Figure 1. Therefore, its later hydrolysis produces the Strecker aldehyde and the modified lipid oxidation product shown in Figure 2. However, imine D is not produced by any of the mechanisms described previously. The difference between this imine and the imines formed by previous mechanisms (Figures 1, 3, and 4) is that the carbon-nitrogen double bond is not at the side of the amino acid residue but at the other side. This structural difference is the responsible for the formation of the amine of the amino acid after hydrolysis. At the same time that the amine is produced,

245 the initial lipid-derived reactive carbonyl is recovered. Therefore, by means of this 246 reaction pathway, amines and Strecker aldehydes of amino acids are produced 247 simultaneously, although the relative amounts at which both compounds are produced are not necessarily the same and it will depend on the amino acid and the lipid-derived reactive carbonyl involved, and the reaction conditions (Zamora et al., unpublished results). 
This mechanism was confirmed by using deuterated water in the reaction (Hidalgo et

252

al, 2010a). The produced amines were mono- and di-deuterated at the $\alpha$-carbon of the amino group. The deuteration of the monodeuterated amine occurred during the 1,2prototropic shift in the azomethine ylide. The second deuterium in the dideuterated amine was introduced at the beginning of the reaction by keto-enol tautomerism in the original amino acid.

\section{CONVERSION OF THE AMINES DERIVED FROM AMINO ACIDS INTO}

\section{STRECKER ALDEHYDES BY LIPID OXIDATION PRODUCTS}

The amines produced in the degradation of amino acids can suffer later reactions in the presence of lipid-derived reactive carbonyls. One of them is their conversion into the corresponding Strecker aldehydes. This reaction, which was observed in the conversion of $\beta$-phenylethylamine into phenylacetaldehyde in the presence of different lipid oxidation products (Zamora et al, 2012b), seems to takes place according to the reaction pathway shown in Figure 7. The initial step is the formation of the corresponding imine between the amino group of the amine and the carbonyl group of the oxidized lipid.

This imine is the same imine D of Figure 6. The conjugated system of the lipid-derived carbonyl and the electronic effects of the substituents at the $\alpha$-carbon of the amine favor an electronic rearrangement that convert imine $\mathrm{D}$ into imine $\mathrm{B}$, which is the same imine B formed in Figures 1 and 6. The hydrolysis of imine B is the origin of the Strecker aldehyde. By means of this reaction, the lipid oxidation product has been transformed into an amino derivative that, in most cases, is the indicated in Figure 2 (Zamora et al., 2012b).

The existence of this reaction suggests that imine D in Figure 6 can be converted into imine B to some extent in the course of the reaction. Therefore, the amine/aldehyde 
ratio produced by pathway collected in Figure 6 is not only influenced by the conversion of the azomethine ylide into one or other imine but also by the conversion of imine $\mathrm{D}$ into imine $\mathrm{B}$. To our best knowledge, the relative contribution of both reactions to the amounts of amines and aldehydes formed by amino acid degradation in the presence of lipid oxidation products has not been investigated at present.

\section{CONVERSION OF THE AMINES INTO VINYLOGOUS DERIVATIVES OF}

\section{AMINO ACIDS BY LIPID OXIDATION PRODUCTS}

$$
\text { In addition to their conversion into Strecker aldehydes, the amines can also suffer an }
$$

elimination reaction to be converted into olefins: the corresponding vinylogous derivatives of the amino acids. The role of oxidized lipids in this reaction was firstly observed in the conversion of phenylalanine into styrene (Hidalgo and Zamora, 2007), and, then, described in detail for the elimination of 3-aminopropionamide to produce acrylamide (Zamora et al., 2009).

The reaction takes place as shown in Figure 8. Alkylamines are usually eliminated through their conversion into quaternary ammonium salts followed by Hofmann elimination (Saunders and Cockerill, 1973). However, in the presence of the lipidderived reactive carbonyl, the amine produces firstly the corresponding imine (imine D). This imine may be then converted into an iminium ion that suffer a milder elimination (Katritzky and El-Mouafy, 1982). This conversion is favored by the lipid because a reactive carbon in the lipid chain may react with the nitrogen of the imine to produce the corresponding iminium ion. Thus, when the reaction was carried out in the presence of alkadienals, the lipid was converted into 2-alkylpyridine, which facilitated the elimination of the amine. 
This reaction pathway was confirmed by determining the activation energies of the elimination reaction of amines, amines in the presence of lipid-derived reactive carbonyls, and $N$-substituted alkylamines. Thus, the elimination reaction of $N$ substituted alkylamines, which took place through iminium ions, had a similar activation energy that the elimination reaction of primary amines in the presence of lipid-derived reactive carbonyls (Zamora et al., 2009). On the other hand, the elimination reaction of primary amines, which can only take place through the formation of ammonium salts, had much higher activation energy. In addition, this mechanism was also in agreement with the reactivities exhibited by the different carbonyl compounds, which were related to the conjugation of the iminium ion.

\section{THE ROLE OF LIPID OXIDATION PRODUCTS ON THE ADDITION OF NUCLEOPHILES TO THE VINYLOGOUS DERIVATIVES OF AMINO ACIDS}

The vinylogous derivatives of amino acids described in the previous section are not stable compounds and can suffer the addition of nucleophiles. This reaction has recently received a considerable attention because of its possible use in the elimination of acrylamide (Adams et al., 2010; Claeys et al., 2005; Kim et al., 2005; Rydberg et al., 2003; Salazar et al., 2012; Zamora et al., 2011a). Although compounds having either amino or sulfhydryl groups are added to these olefins, there are significant differences between both kinds of compounds.

The addition of amino compounds takes place as indicated in Figure 9. The vinylogous derivative of the amino acid reacts very rapidly and easily with the amino compound to produce the corresponding Michael adduct (adduct A). However, this adduct still has a nucleophilic group and it can react with a new molecule of olefin to produce a new adduct (adduct B). Nevertheless, adduct B is not usually produced under 
usual reaction conditions because vinylogous derivatives of amino acids are produced to

a much lower extent than the amount at which amino compounds are present (Zamora et al., 2010). Both additions are reversible and it is possible to recover the initial olefin just by heating the adduct (Zamora et al., 2010).

When lipid-derived reactive carbonyls are present, this Michael addition is inhibited, most likely as a consequence of the reaction of the carbonyl compound with the amino compound to produce the corresponding imine (imine E). This reaction avoids that the amino compound can react with the olefin. This competition was confirmed when eliminate acrylamide. Although cysteine is much more effective than $N$-acetylcysteine, when a carbonyl compound was present, the effectiveness of both compounds was very similar (Zamora et al., 2011b).

Differently to the addition of amino compounds, the addition of compounds having a sulfhydryl group is not an equilibrium and the atmospheric oxygen plays a role. The reaction follows the reaction pathway shown in Figure 10. produce the corresponding adduct (adduct C). This reaction has an activation energy more easily than the addition of amino compounds.

When oxygen is present, thiols are also converted into the corresponding thiolyl radicals which can either polymerize or be added to the olefin to form a new radical that will continue the free radical chain. This alternative route was confirmed by inhibition of free radical reactions with antioxidants (Hidalgo et al., 2010b). 
To our best knowledge, the effect of carbonyls in this reaction has not been studied so far. However, lipid-derived reactive carbonyls usually have a conjugated system that might suffer the addition of the thiol. The role that this alternative reaction may play in the removal of the olefins produced during amino acid degradation remains to be investigated.

\section{COMPETITION AMONG THE DIFFERENT ROUTES OF AMINO ACID}

\section{DEGRADATION PRODUCED BY LIPID OXIDATION PRODUCTS}

As described above, amino acids can be degraded by a variety of pathways that produce many different products. However, all these routes are interconnected and the products of some reactions are frequently reactants of new reactions. Figure 11 shows schematically how all the routes discussed previously are interconnected. As can be observed, in the presence of lipid-derived reactive carbonyls, amino acids always produce the corresponding imine in the first step (imine A). This imine suffers then an electronic rearrangement to be converted into imines $\mathrm{B}, \mathrm{C}$, or $\mathrm{D}$, depending on what is produced: the exit of the carboxylic proton, the exit of the proton at the $\alpha$-carbon of the amino acid, or the formation of an intermediate 5-oxazolidinone, respectively.

Nevertheless, the azomethine ylide formed by decarboxylation of the 5-oxazolidinone is also responsible for the formation of imine $\mathrm{B}$, and imine $\mathrm{D}$ can be converted into imine products: Strecker aldehydes, $\alpha$-keto acids, and amines, respectively. However, none of these primary amino acid degradation products is a final compound and they may suffer further reactions. Thus, $\alpha$-keto acids can be both decarboxylated to produce the Strecker aldehyde and oxidized to the corresponding aldehyde having two carbons less than the initial amino acid. This oxidation product can also be produced by oxidation of the 
370 Strecker aldehyde. Finally, the amine can be either converted into the Strecker aldehyde

371 or suffer an elimination reaction to produce the corresponding vinylogous derivative of

372 the amino acid. This last olefin is also not a final compound and may suffer the

373 additions of nucleophiles. In the case of amino compounds, the main product that

374 should be expected under usual reaction conditions is adduct A. In the case of thiols,

375 both the formation of adduct $\mathrm{C}$ and, if the reaction is carried out under oxidative

376 conditions, free radical reactions should be expected.

377 Although all these reactions can be produced simultaneously, some of them will be

378 favored over others depending on the reaction conditions and the amino acids and lipid-

379 derived reactive carbonyls involved. In addition, the relative proportions in which the

380 different products are formed will play a major role on the quality of the food product

381 because both food flavors and potentially toxic compounds are formed at the same time

382 that essential amino acids are destroyed (Capuano and Fogliano, 2011; Jackson, 2009;

383 van Boekel et al., 2010). Therefore, it is very important to know how some reactions

384 could be favored over others in order to increase the amount of beneficial products

385 formed and to reduce the amount of non-desirable products.

386 Activation Energy

387 Activation energy is defined as the minimum energy required to start a chemical reaction. Therefore, the comparison among the activation energies of the different reactions in Figure 11 may help to understand what reactions will be firstly produced. The activation energies determined for some of the reactions included in Figure 11 are collected in Table 1. As can be observed, both amino acids and carbonyls usually play a

392 significant role in the activation energy of the reaction. For example, alkadienals are better than other lipid oxidation products for producing the conversion of phenylalanine 
into phenylacetaldehyde. However, there is not any difference between alkadienals and oxoalkenals for converting phenylethylamine into phenylacetaldehyde.

The role of the amino acid can be observed in the conversion of the amino acid into the amine. As observed in Table 1 the activation energy for the conversion of phenylalanine into phenylethylamine is much lower than the conversion of asparagine into 3-aminopropionamide. Therefore, the decarboxylation of phenylalanine should be expected to take place under softer reaction conditions than the decarboxylation of asparagine, which is agreement with the strong heating conditions required for the formation of acrylamide (Tareke et al., 2002).

In addition to the role of amino acids and lipid oxidation products, the different routes shown in Figure 11 have different activation energies. Thus, the activation energy of the conversion of phenylalanine into phenylacetaldehyde is slightly higher than the conversion of phenylalanine into phenylpyruvic acid. However, the conversion of phenylalanine into phenylethylamine seems to require a higher energy. This also happens when two reactions are liked. Thus, higher activation energy is required to convert asparagine into 3-aminopropionamide than to transform 3-aminopropionamide into acrylamide. But the formation of the adduct between acrylamide and $\mathrm{N}$ acetylcysteine still has a lower activation energy. Therefore, from an energy activation point of view, once the decarboxylation is produced, the reaction should finished in the adduct if a nucleophile having a sulhydryl group is present. In practice, this is not completely true, because reaction conditions also play a major role in these reactions.

\section{Effect of $p H$}

Different studies have shown that formation of Strecker aldehydes, $\alpha$-keto acids, amines, and the conversion of amines into Strecker aldehydes are favored at acid pHs 
418 (Hidalgo et al., 2005; 2010a; Zamora et al., 2006b; 2012a; 2012b). On the contrary, the

419

420

421

422

423

424

425

426

427

428

429

430

431

432

433

434

435

436

437

438

439

440

conversion of 3-aminopropionamide into acrylamide or the addition of nucleophiles to acrylamide takes place better at neutral or slightly basic pHs (Hidalgo et al., 2010b;

Zamora et al., 2009).

\section{Effect of water activity}

Water activity is also important. In particular, the elimination reaction to produce olefins from amines is very sensitive to water (Zamora et al., 2009). For that reason, the effect of water activity on the formation of acrylamide from asparagine (Hidalgo et al., 2009) is a compromise between the water activities required for the decarboxylation reaction (Hidalgo et al., 2010a) and for the elimination reaction (Zamora et al., 2009).

\section{Effect of oxygen}

The amount of oxygen also plays a major role on the products formed because free radical reactions are favored. For that reason, Strecker degradations are favored (Hidalgo and Zamora 2007). In addition, the presence of oxygen also favors the conversion of amines into Strecker aldehydes (Zamora et al., 2012b) or the formation of shorter aldehydes from Strecker aldehydes or $\alpha$-keto acids (Zamora et al., unpublished).

On the contrary, the formation of olefins is inhibited in the presence of oxygen (Hidalgo and Zamora, 2007; Hidalgo et al., 2009).

\section{CONCLUSIONS}

The above results show that amino acids are easily degraded by lipid oxidation products in a similar way to carbohydrates. According to the data published to date, this degradation produces three kinds of compounds in a first step: Strecker aldehydes, $\alpha-$ keto acids, and amines. However, they are not final compounds and these compounds 
441 are involved in further reactions. Thus, $\alpha$-keto acids are transformed into Strecker

442 aldehydes and other aldehydes having two carbons less than the original amino acid; the

443 amines are transformed into Strecker aldehydes and olefins; and the Strecker aldehydes

444 can be oxidized to shorter aldehydes. Finally, olefins are susceptible to suffer addition

445 of nucleophilic compounds. Although all these reactions are interconnected, it is

446 possible to modify the ratio among the different formed products as a function of the

447 oxidized lipid and the amino acid involved, and the reaction conditions (particularly,

$448 \mathrm{pH}$, water activity, presence of oxygen, time, and temperature). By playing with these

449 tools it is possible to favor the formation of the desirable compounds produced in these

450 reactions and to decrease the amount of the undesired products that are also formed.

451 Further studies on how benefit/risk balance of amino acid degradations by lipid

452 oxidation products can be improved upon processing on actual food systems are needed.

453 ACKNOWLEDGEMENT

454 We are indebted to José L. Navarro for technical assistance. This study was

455 supported in part by the European Union (FEDER funds) and the Plan Nacional de I +

456 D of the Ministerio de Economía y Competitividad of Spain (projects AGL2009-07638

457 and AGL2012-35627). 
460

461

462

463

464

465

466

467

468

469

470

471

472

473

474

475

476

477

478

479

480

Adams, A., Hamdani, S., Van Lancker, F., Mejri, S., De Kimpe, N. (2010). Stability of acrylamide in model systems and its reactivity with selected nucleophiles. Food Res. Int. 43: 1517-1522.

Ardö, Y. (2006). Flavour formation by amino acid catabolism. Biotechnol. Adv. 24: 238-242.

Aurelio, L., Box, J. S., Brownlee, R. T. C., Hughes, A. B., Sleebs, M M. (2003). An efficient synthesis of $N$-methyl amino acids by way of intermediate 5oxazolidinones. J. Org. Chem. 68: 2652-2667.

Capuano, E., Fogliano, V. (2011). Acrylamide and 5-hydroxymethylfurfural (HMF): A review on metabolism, toxicity, occurrence in food and mitigation strategies. LWTFood Sci. Technol. 44: 793-810.

Capuano, E., Oliviero, T., Acar, O. C., Gokmen, V., Fogliano, V. (2010). Lipid oxidation promotes acrylamide formation in fat-rich model systems. Food Res. Int. 43: 1021-1026.

Cheng, H. F. (2010). Volatile flavor compounds in yogurt: A review. Crit. Rev. Food Sci. Nutr. 50: 938-950.

Choe, E., Min, D. B. (2006). Chemistry and reactions of reactive oxygen species in foods. Crit. Rev. Food Sci. Nutr. 46: 1-22.

Chu, F. L., Yaylayan, V. A. (2008). Model studies on the oxygen-induced formation of benzaldehyde from phenylacetaldehyde using pyrolysis GC-MS and FTIR. J. Agric. Food Chem. 56: 10697-10704. 
481 Chu, F. L., Yaylayan, V. A. (2009). FTIR monitoring of oxazolidin-5-one formation 482 and decomposition in a glycolaldehyde-phenylalanine model system by isotope 483 labeling techniques. Carbohydr. Res. 344: 229-236.

484

485

486

487

488

489

490

491

492

493

494

495

496

497

498

499

500

501

502

Claeys, W. L., De Vleeschouwer, K., Hendrickx, M. E. (2005). Effect of amino acids on acrylamide formation and elimination kinetics. Biotechnol. Prog. 21: 1525-1530.

Fernández, M., Zúñiga, M. (2006). Amino acid catabolic pathways of lactic acid bacteria. Crit. Rev. Microbiol. 32: 155-183.

Fürmeier, S., Metzger, J. O. (2003). Synthesis of new heterocyclic fatty compounds. Eur. J. Org. Chem.: 885-893.

Gardner, H. W. (1989). Oxygen radical chemistry of polyunsaturated fatty acids. Free Radical Biol. Med. 7: 65-86.

Gardner, H. W., Hou, C. T., Weisleder, D., Brown, W. (2000). Biotransformation of linoleic acid by Clavibacter sp ALA2: Heterocyclic and heterobicyclic fatty acids. Lipids 35: 1055-1060.

Girotti, A. W. (1998). Lipid hydroperoxide generation, turnover, and effector action in biological systems. J. Lipid Res. 39: 1529-1542.

Granvogl, M., Beksan, E., Schieberle, P. (2012). New insights into the formation of aroma-active Strecker aldehydes from 3-oxazolines as transient intermediates. $J$. Agric. Food Chem. 60: 6312-6322.

Granvogl, M., Bugan, S., Schieberle, P. (2006). Formation of amines and aldehydes from parent amino acids during thermal processing of cocoa and model systems: new insights into pathways of the Strecker reaction. J. Agric. Food Chem. 54: 1730-1739. 
503 Grosch, W. (1976). Degradation of linoleic acid hydroperoxides to volatile carbonyl $504 \quad$ compounds. Z. Lebensm. Unters. Forsch. 160: 371-375.

505 Hidalgo, F. J., Delgado, R. M., Navarro, J. L., Zamora, R. (2010a). Asparagine 506 decarboxylation by lipid oxidation products in model systems. J. Agric. Food Chem. $507 \quad$ 58: 10512-10517.

508

509

510

511

512

513

514

515

516

517

518

519

520

521

522

523

Hidalgo, F. J., Delgado, R. M., Zamora, R. (2009). Degradation of asparagine to acrylamide by carbonyl-amine reactions initiated by alkadienals. Food Chem. 116: 779-784.

Hidalgo, F. J., Delgado, R. M., Zamora, R. (2010b). Role of mercaptans on acrylamide elimination. Food Chem. 122: 596-601.

Hidalgo, F. J., Gallardo, E., Zamora, R. (2005). Strecker type degradation of phenylalanine by 4-hydroxy-2-nonenal in model systems. J. Agric. Food Chem. 54(53): 10254-10259.

Hidalgo, F. J., Zamora, R. (1995). In vitro production of long chain pyrrole fatty esters from carbonyl-amine reactions. J. Lipid Res. 36: 725-735.

Hidalgo, F. J., Zamora, R. (2004). Strecker-type degradation produced by the lipid oxidation products 4,5-epoxy-2-alkenals. J. Agric. Food Chem. 52: 7126-7131.

Hidalgo, F. J., Zamora, R. (2007). Conversion of phenylalanine into styrene by 2,4decadienal in model systems. J. Agric. Food Chem. 55: 4902-4906.

Jackson, L. S. (2009). Chemical food safety issues in the United States: Past, present, and future. J. Agric. Food Chem. 57: 8161-8170. 
524 Kaneda, K., Itoh, T., Kii, N., Jitsukawa, K., Teranishi, S. (1982). Oxygenation of 525 enamines using copper catalysts. J. Mol. Catal. 15: 349-365.

526 Kasaikina, O. T., Kansheva, V. D., Maximova, T. V., Kartasheva, Z. S., Yanishlieva, N.

527 V., Kondratovich, V. G., Totseva, I. R. (2006). Catalytic effect of amphiphilic

528

529

530

531

532

533

534

535

536

537

538

539

540

541

542

543

544

545

Kim, C. T., Hwang, E.-S., Lee, H. J. (2005). Reducing acrylamide in fried snack products by adding amino acids. J. Food Sci. 70: C354-C358.

Marcobal, A., de las Rivas, B., Landete, J. M., Tabera, L., Muñoz, R. (2012). Tyramine and phenylethylamine biosynthesis by food bacteria. Crit. Rev. Food Sci. Nutr. 52: $448-467$.

Pazos, M., Andersen, M. L., Skibsted, L. H. (2008). Heme-mediated production of free radicals via performed lipid hydroperoxide fragmentation. J. Agric. Food Chem. 56: $11478-11484$.

Reis, A., Spickett, C. M. (2012). Chemistry of phospholipid oxidation. Biochim. Biophis. Acta 1818: 2374-2387.

Rydberg, P., Eriksson, S., Tareke, E., Karlsson, P., Ehrenberg, L., Törnqvist, M. (2003). Investigations of factors that influence the acrylamide content of heated foodstuffs. $J$. Agric. Food Chem. 51: 7012-7018. 
546 Salazar, R., Arámbula-Villa, G., Vázquez-Landaverde, P. A., Hidalgo, F. J., Zamora, R. 547 (2012). Mitigating effect of amaranth (Amarantus hypochondriacus) protein on $548 \quad$ acrylamide formation in foods. Food Chem. 135: 2293-2298.

Saunders, W. H., Cockerill, A. F. (1973). Mechanisms of Elimination Reactions. Wiley, 550 New York.

Shalaby, A. R. (1996). Significance of biogenic amines to food safety and human health. Food Res. Int. 29: 675-690.

Smit, B. A., Engels, W. J. M., Alewijm, M., Lommerse, G. T. C. A., Kippersluijs, E. A.

Taeymans, D., Wood, J., Ashby, P., Blank, I., Studer, A., Stadler, R. H., Gonde, P., Van Eijck, P., Lalljie, S., Lingnert, H., Lindblom, M., Matissek, R., Muller, D., Tallmadge, D., O'Brien, J., Thompson, S., Silvani, D., Whitmore, T. (2004). A review of acrylamide: An industry perspective on research, analysis, formation and control. Crit. Rev. Food Sci. Nutr. 44: 323-347.

Tareke, E., Rydberg, P., Karlsson, P., Eriksson, S., Törnqvist, M. (2002). Analysis of acrylamide, a carcinogen formed in heated foodstuffs. J. Agric. Food Chem. 50: 4998-5006.

Tokunaga, M., Shirogane, Y., Aoyama, H., Obora, Y., Tsuji, Y. Copper-catalyzed oxidative cleavage of carbon-carbon double bond of enol ethers with molecular oxygen. J. Organomet. Chem. 690: 5378-5382. 
Tsuge, O., Kanemasa, S., Ohe, M., Takenaka, S. (1987). Simple generation of nonstabilized azomethine ylides through decarboxylative condensation of $\alpha$-amino acids with carbonyl-compounds via 5-oxazolidinone intermediates. Bull. Chem. Soc. Jpn. 60: 4079-4089.

Ueda, J., Saito, N., Ozawa, T. (1996). Detection of free radicals produced from reactions of lipid hydroperoxide model compounds with $\mathrm{Cu}(\mathrm{II})$ complexes by ESR spectroscopy. Arch. Biochem. Biophys. 325: 65-76.

Urbach, G. (1993). Relations between cheese flavor and chemical composition. Int. Dairy J. 3: 389-422.

Van Boekel, M., Fogliano, V., Pellegrini, N., Stanton, C., Scholz, G., Lalljie, S., Somoza, V., Knorr, D., Jasti, P. R., Eisenbrand, G. (2010). A review on the beneficial aspects of food processing. Mol. Nutr. Food Res. 54: 1215-1247.

Yaylayan, V. A., Keyhani, A. (2001). Carbohydrate and amino acid degradation pathways in L-methionine/D- $\left[{ }^{13}\right.$ C]glucose model systems. J. Agric. Food Chem. 49: 800-803.

Zamora, R., Delgado, R. M., Hidalgo, F. J. (2009). Conversion of 3-aminopropionamide and 3-alkylaminopropionamides into acrylamide in model systems. Mol. Nutr. Food Res. 53: 1512-1520.

Zamora, R., Delgado, R. M., Hidalgo, F. J. (2010). Model reactions of acrylamide with selected amino compounds. J. Agric. Food Chem. 58: 1708-1713.

Zamora, R., Delgado, R. M., Hidalgo, F. J. (2011a). Amino phospholipids and lecithins as mitigating agents for acrylamide in asparagine/glucose and asparagine/2,4decadienal model systems. Food Chem. 126: 104-108. 
Zamora, R., Delgado, R. M., Hidalgo, F. J. (2011b). Strecker aldehydes and $\alpha$-keto acids, produced by carbonyl-amine reactions, contribute to the formation of acrylamide. Food Chem. 128: 465-470.

Zamora, R., Delgado, R. M., Hidalgo, F. J. (2012a). Formation of $\beta$-phenylethylamine as a consequence of lipid oxidation. Food Res. Int. 46: 321-325.

Zamora, R., Delgado, R. M., Hidalgo, F. J. (2012b). Chemical conversion of phenylethylamine into phenylacetaldehyde by carbonyl-amine reactions in model systems. J. Agric. Food Chem. 60: 5491-5496.

Zamora, R., Gallardo, E., Hidalgo, F. J. (2006a). Amine degradation by 4,5-epoxy-2decenal in model systems. J. Agric. Food Chem. 54: 2398-2404.

Zamora, R., Gallardo, E., Hidalgo, F. J. (2007). Strecker degradation of phenylalanine initiated by 2,4-decadienal or methyl 13-oxooctadeca-9,11-dienoate in model systems. J. Agric. Food Chem. 55: 1308-1314.

Zamora, R., Gallardo, E., Hidalgo, F. J. (2008). Model studies on the degradation of phenylalanine initiated by lipid hydroperoxides and their secondary and tertiary oxidation products. J. Agric. Food Chem. 56: 7970-7975.

Zamora, R., Gallardo, E., Navarro, J. L., Hidalgo, F. J. (2005). Strecker-type degradation of phenylalanine by methyl 9,10-epoxy-13-oxo-11-octadecenoate and methyl 12,13-epoxy-9-oxo-11-octadecenoate. J. Agric. Food Chem. 53: 4583-4588.

Zamora, R., Hidalgo, F. J. (2005). Coordinate contribution of lipid oxidation and Maillard reaction to the nonenzymatic food browning. Crit. Rev. Food Sci. Nutr. 45: 49-59. 
Zamora, R., Hidalgo, F. J. (2008). Contribution of lipid oxidation products to

614 acrylamide formation in model systems. J. Agric. Food Chem. 56: 6075-6080.

615 Zamora, R., Navarro, J. L., Gallardo, E., Hidalgo, F. J. (2006b). Chemical conversion of

$616 \alpha$-amino acids into $\alpha$-keto acids by 4,5-epoxy-2-decenal. J. Agric. Food Chem. 54:

$617 \quad 6101-6105$.

618 Zhang, Y., Ren, Y., Zhang, Y. (2009). New research developments on acrylamide:

619 analytical chemistry, formation mechanism, and mitigation recipes. Chem. Rev.

$620 \quad$ 2009: 4375-4397.

621 
623 Figure 1. Proposed pathway for the conversion of amino acids into Strecker aldehydes in 624 the presence of lipid-derived reactive carbonyls.

625 Figure 2. Transformations suffered by lipid-derived reactive carbonyls in the Strecker 626 degradation of amino acids.

627 Figure 3. Proposed pathway for the free radical degradation of amino acids.

628 Figure 4. Proposed pathway for the conversion of amino acids into $\alpha$-keto acids in the 629 presence of lipid-derived reactive carbonyls.

630 Figure 5. Proposed pathways for the conversion of $\alpha$-keto acids and Strecker aldehydes 631 into shorter aldehydes under oxidative conditions.

632 Figure 6. Proposed pathway for the conversion of amino acids into amines and Strecker 633 aldehydes in the presence of lipid-derived reactive carbonyls.

634 Figure 7. Proposed pathway for the conversion of amines into Strecker aldehydes in the 635 presence of lipid-derived reactive carbonyls.

636 Figure 8. Proposed pathway for the conversion of amines into olefins in the presence of 637 lipid-derived reactive carbonyls.

638 Figure 9. Reaction of olefins with amino compounds and role of lipid-derived reactive 639 carbonyls in these reactions.

640 Figure 10. Reactions of olefins with thiols.

641 Figure 11. General scheme for amino acid degradations induced by lipid-derived reactive 642 carbonyls. 
Table 1 Activation energies of amino acid degradation reactions produced by lipid-derived reactive carbonyls ${ }^{\mathrm{a}}$

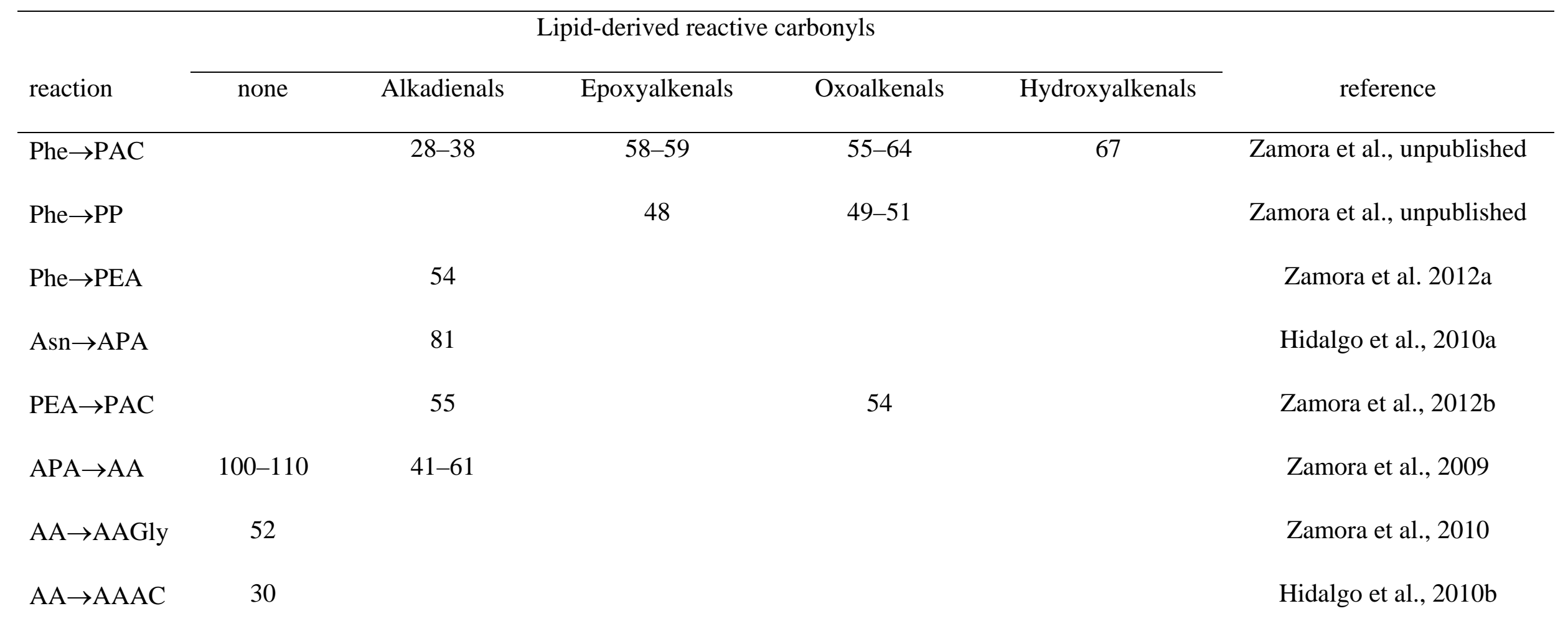

${ }^{\text {a}}$ Values are given in kJ/mol. Abbreviations: AA, acrylamide; AAAC, adduct between acrylamide and $N$-acetylcysteine; AAGly, adduct between acrylamide and glycine; APA, 3-aminopropionamide; Asn, asparagine; PAC, phenylacetaldehyde; PEA, phenylethylamine; Phe, phenylalanine; PP, phenylpyruvic acid. 


$$
\text { (N) }
$$

Figure 1 
short-chain lipid oxidation products

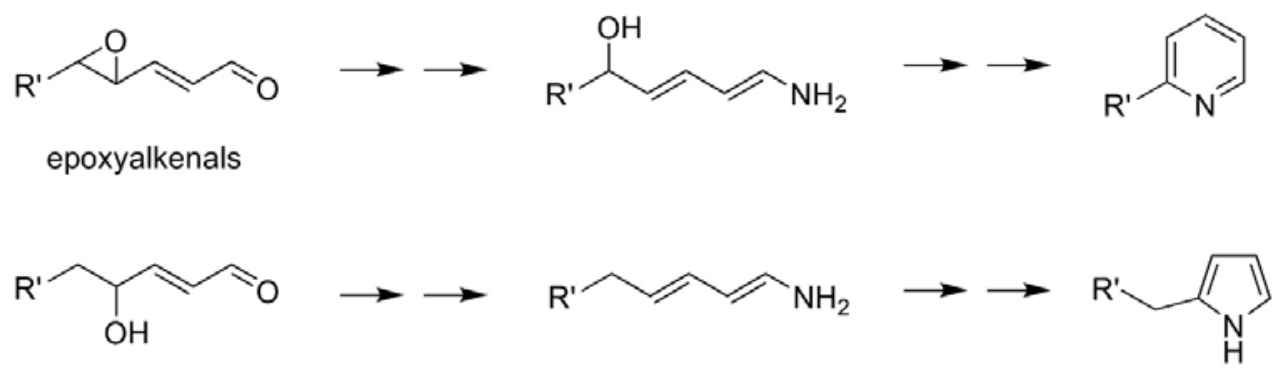

hydroxyalkenals<smiles>[R]CC(=O)/C=C/C=O</smiles>

oxoalkenals

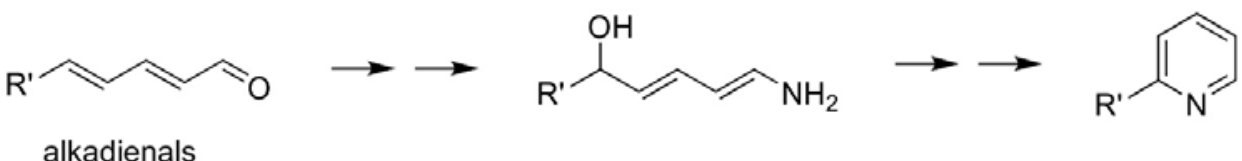

long-chain lipid oxidation products

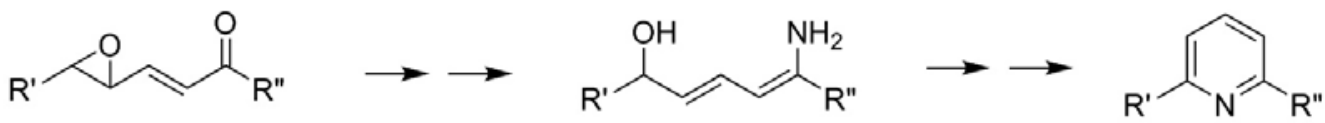

epoxyoxooctadecenoates<smiles>[R]C=CC=CC([R])=O</smiles><smiles>CCCCC</smiles><smiles>[R]C(N)=CC=CC([R])O</smiles><smiles>C=CCCC</smiles><smiles>[R]c1cccc([R])n1</smiles>

oxooctadecadienoates

Figure 2 

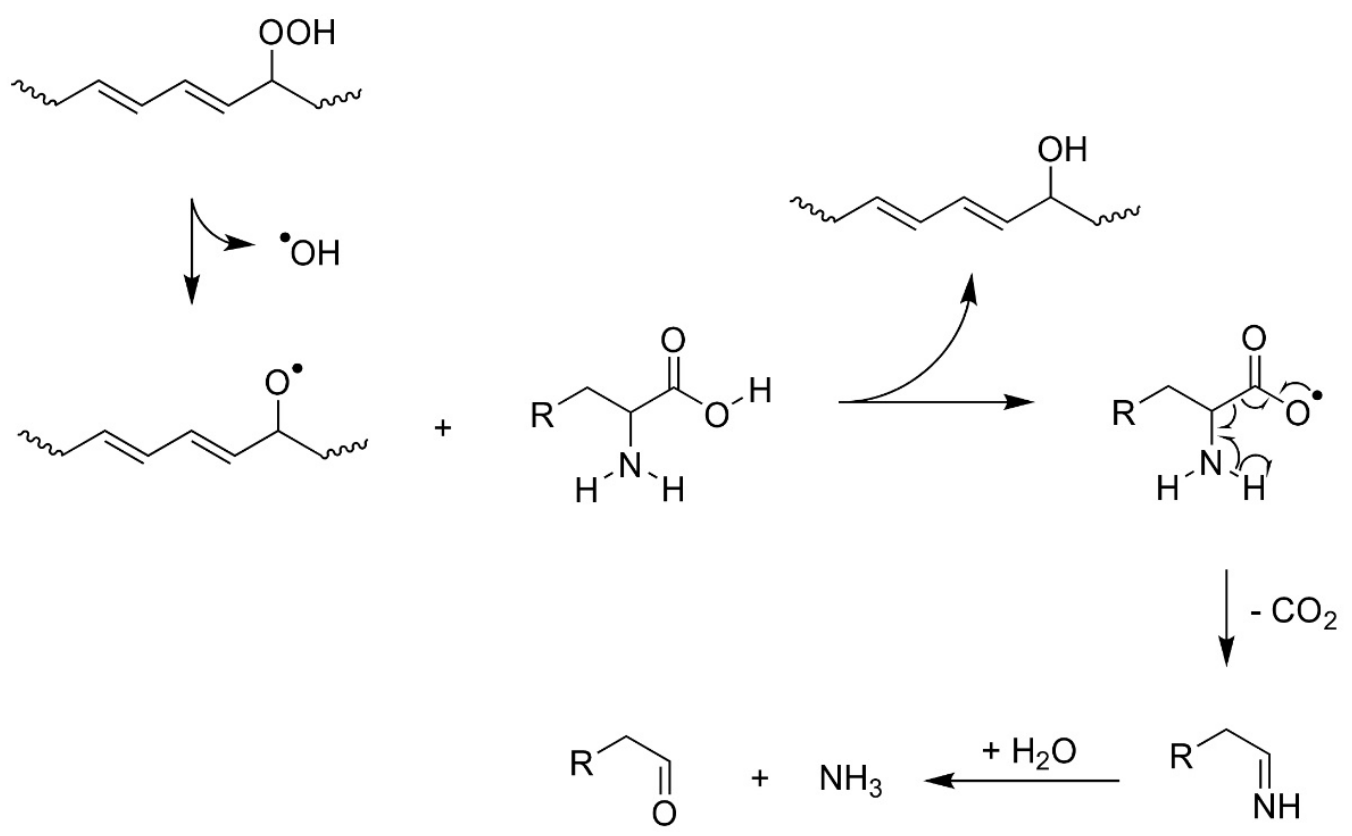

Figure 3 
$\overbrace{\mathrm{NH}_{2}}^{\mathrm{O}} \mathrm{O}^{-\mathrm{H}}$$$
\text { 型 }
$$<smiles>CCCCC/C=C/C=C/C=O</smiles>

$\stackrel{-\mathrm{H}_{2} \mathrm{O}}{\longrightarrow}$

$\overbrace{\mathrm{O}}^{\mathrm{O}} \overbrace{\mathrm{O}^{-H}}^{\mathrm{H}}+$<smiles>C/C=C/C=C/N</smiles>

$\longleftarrow+\mathrm{H}_{2} \mathrm{O}$

imine C

Figure 4 


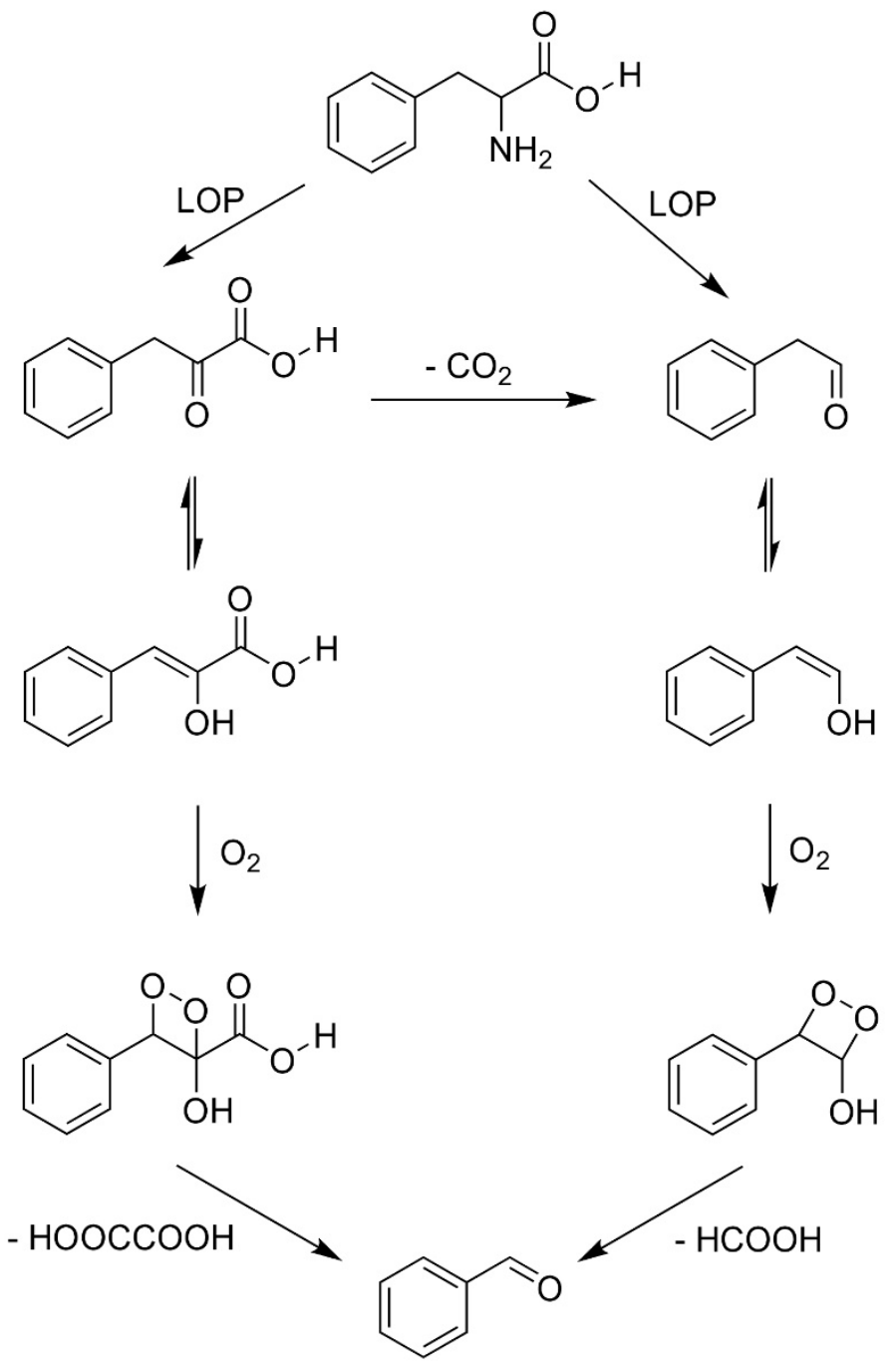

Figure 5 


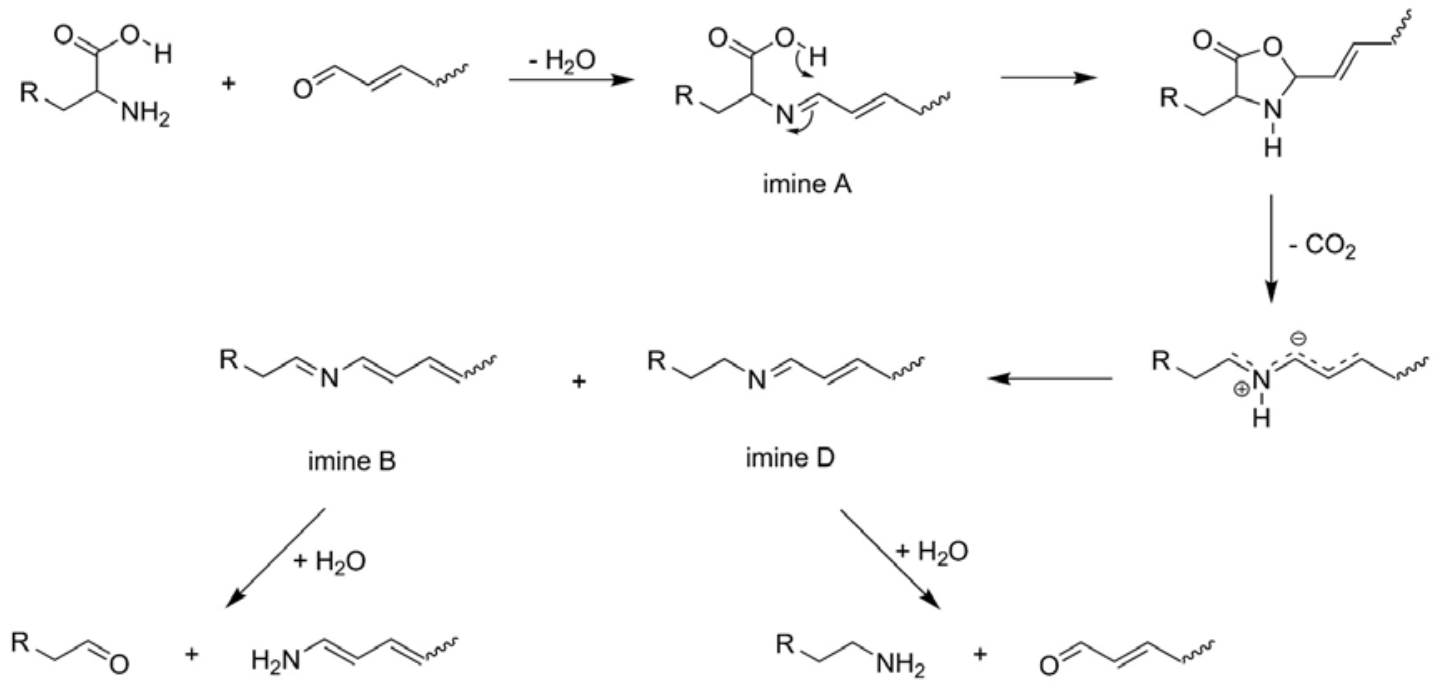

Figure 6 


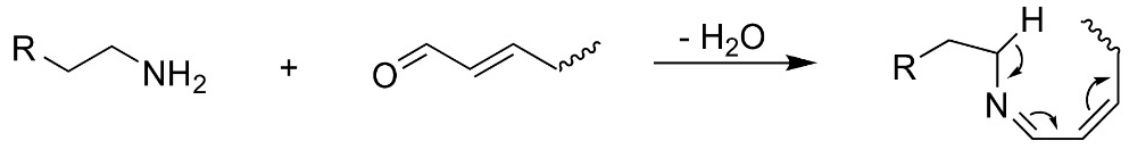

$$
\begin{aligned}
& \text { imine D } \\
& \downarrow
\end{aligned}
$$

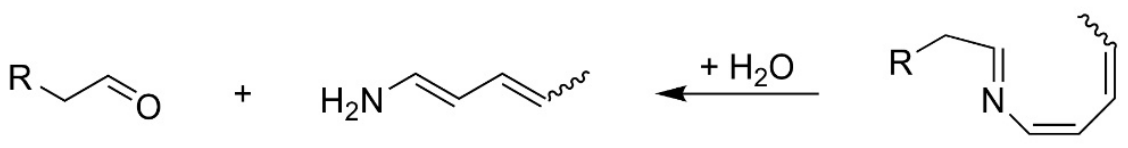

$$
\begin{aligned}
& \text { imine B }
\end{aligned}
$$

Figure 7 


$$
\mathrm{NH}_{2}+\mathrm{N}_{\mathrm{m}}^{\mathrm{N}} \stackrel{-\mathrm{H}_{2} \mathrm{O}}{\longrightarrow}
$$

Figure 8 


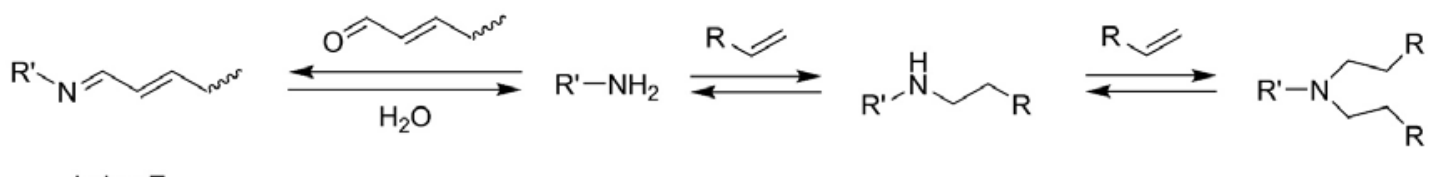

$$
\begin{aligned}
& \text { imine } \mathrm{E} \text { adduct } \mathrm{A} \text { adduct } \mathrm{B}
\end{aligned}
$$

Figure 9 


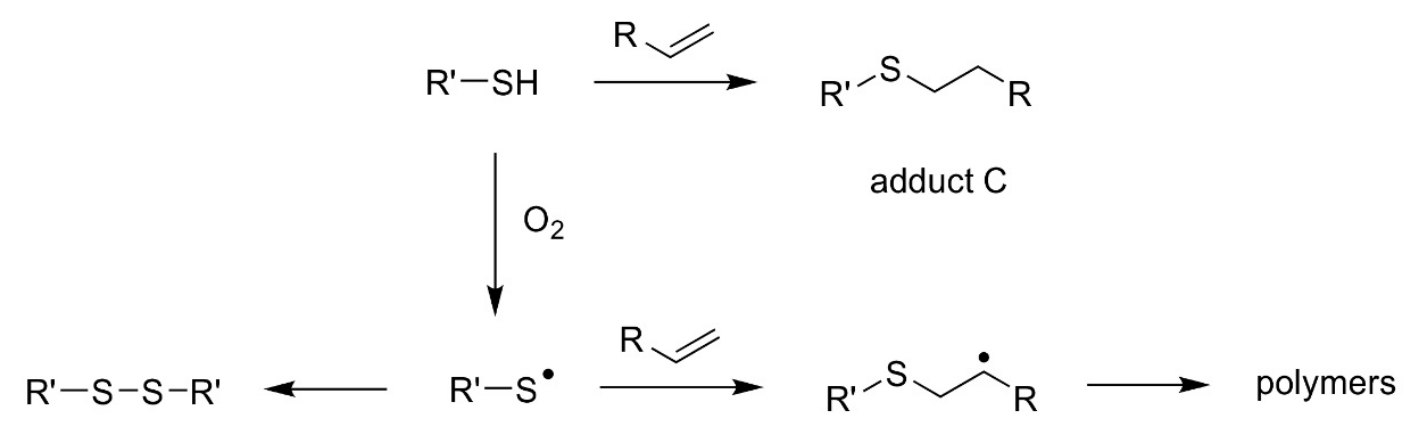

Figure 10 


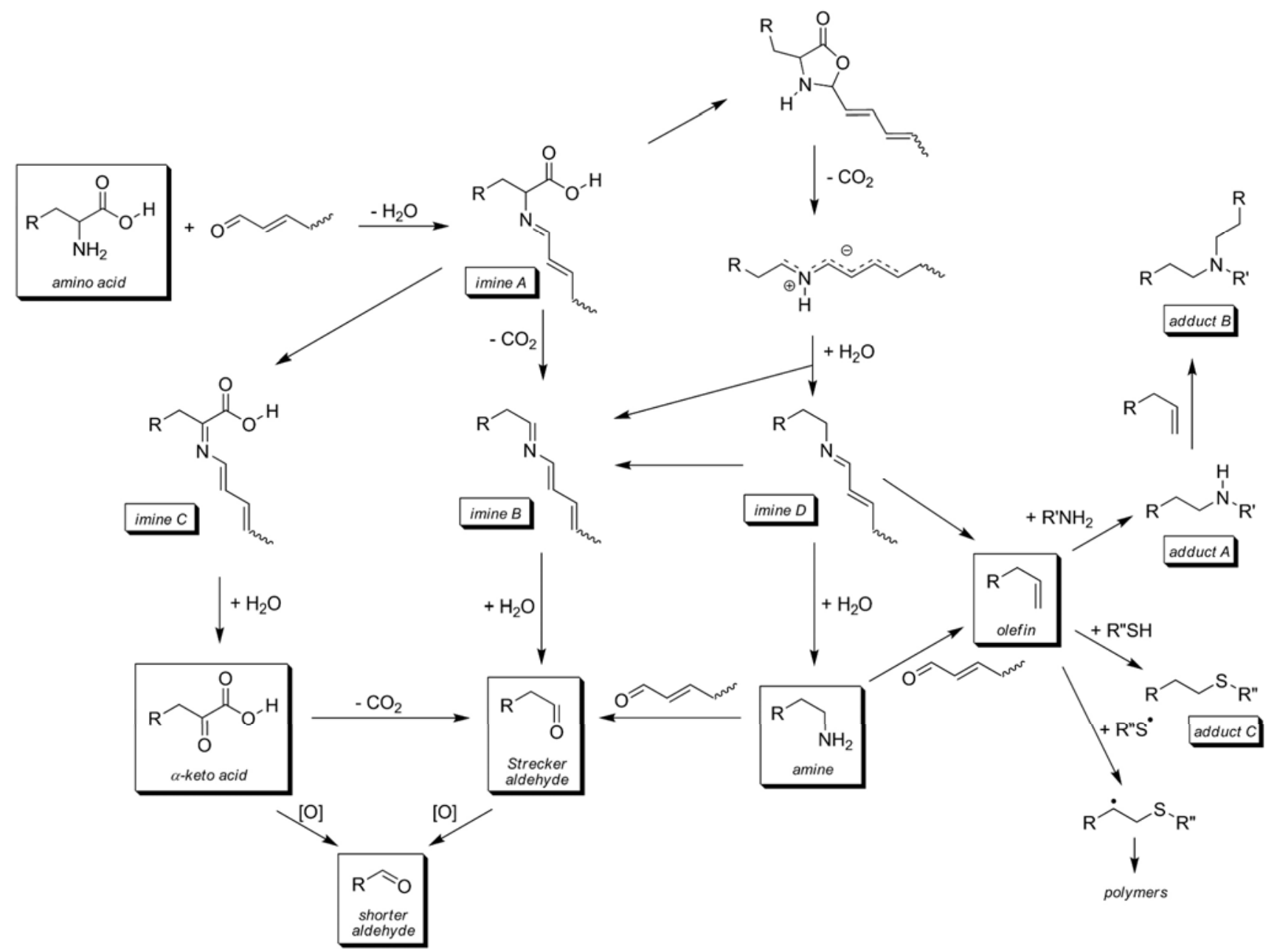

Figure 11 